\title{
DETERMINANTES SOCIODEMOGRÁFICOS Y LA VIOLENCIA CONTRA LA MUJER. TACNA PERÚ: UN ANÁLISIS RETROSPECTIVO DE LOS DATOS DE LOS CENTROS DE EMERGENCIA MUJER
}

\author{
Olga Choque Chura ${ }^{1, a}$, Rinna Myriam Pilco Velasquez ${ }^{1, b}$, Jackeline Flores Flores ${ }^{1, c}$, \\ Liliana Angélica De La Macarena Rivas Hidalgo ${ }^{1, d}$.
}

\begin{abstract}
RESUMEN
Objetivos. Determinar la relación de los determinantes sociodemográficos y la violencia contra la mujer. Tacna 2015-2017. Materiales y métodos. Estudio transversal, con una muestra de 1945 casos que realizaron denuncia. El análisis estadístico utilizó el chi- cuadrado, O.R. (razón de prevalencia) para análisis de los determinantes asociados, considerándose un valor p significativo $<0.05$. Resultados. La violencia psicológica es la más predominante. Los determinantes sociodemográficos de la agredida asociados a violencia psicológica y física fueron: edad, estado civil, nivel educativo y condición de trabajo remunerado. Los determinantes de la agredida asociados a violencia sexual son: edad (más vulnerables $<19$ años). Los determinantes del agresor asociadas a violencia psicológica fueron: edad y sexo siendo las mujeres las que realizan mayormente este tipo de violencia; Los determinantes del agresor asociados a violencia física: edades intermedias y convivencia. Los determinantes asociados a violencia sexual son: edad, sexo y la convivencia con la víctima, con un valor $p=<0,001$ para la mayoría de estas asociaciones. Conclusiones. Según los determinantes sociodemográficos de la agredida, a mayor edad mayor riesgo de violencia psicológica, edades entre 20 y 49 años mayor riesgo de violencia física, $<19$ años mayor riesgo de violencia sexual. Tener trabajo remunerado aumenta el riesgo de violencia. Determinantes del agresor predominantes son: edad con características similares para la violencia psicológica, física y sexual que, en la mujer, con evidencia que la mujer agrede a otra mujer.
\end{abstract}

Palabras Clave: Determinantes sociodemográficos; Violencia contra la mujer (Fuente: DeCS BIREME).

\section{SOCIODEMOGRAPHIC DETERMINANTS AND VIOLENCE AGAINST WOMEN. TACNA PERU: A RETROSPECTIVE ANALYSIS OF THE DATA OF THE WOMEN EMERGENCY CENTER}

\begin{abstract}
Objectives. To determine the relationship of sociodemographic determinants and violence against women. Tacna 20152017. Materials and methods. Cross-sectional study, with a sample of 1945 cases that filed a complaint. Statistical analysis used the square, O.R. (prevalence ratio) for analysis of the associated determinants, considering a significant $p$-value $<0.05$. Results. Psychological violence is the most prevalent. The sociodemographic determinants of the assault associated with psychological and physical violence were: age, marital status, educational level and status of paid work. The determinants of the assault associated with sexual violence are: age (most vulnerable $<19$ years). The determinants of the aggressor associated with psychological violence were: age and sex, with women being the ones who mostly carry out this type of violence; The determinants of the aggressor associated with physical violence: intermediate ages and coexistence. The determinants associated with sexual violence are: age, sex and living with the victim, with a value $p=<0.001$ for most of these associations. Conclusions. According to the sociodemographic determinants of the assault, the older people are at greater risk of psychological violence, ages between 20 and 49 years are at higher risk of physical violence, $<19$ years are at higher risk of sexual violence. Having paid work increases the risk of violence. The predominant determinants of the aggressor are: age with similar characteristics for psychological, physical and sexual violence than in women, with evidence that the woman aggravates another woman.
\end{abstract}

Keywords: Sociodemographic determinants; Violence against women (Source: MeSH NLM).

Universidad Nacional Jorge Basadre Grohmann de Tacna, Perú.

Licenciada en Obstetricia-Doctor en Ciencia con mención en Salud Pública-Docente-UNJBG. Tacna, Perú

Licenciada en Obstetricia-Doctor en Ciencia con mención en Salud Pública-Docente-UNJBG. Tacna, Perú

Licenciada en Obstetricia-Profesor-UNJBG. Tacna, Perú.

Licenciada en Psicología-Magister en Psicología clínica y de familia. Psicóloga-UNJBG. Tacna, Perú.

Citar como: Choque O, Pilco RM, Flores J, De La Macarena LA. Determinantes sociodemográficos y la violencia contra la mujer. Tacna Perú: un analisis retrospectivo de los datos de los centro de emergencia mujer. Rev Peru Investig Matern Perinat 2019; 8(3):34-9 DOI https://doi.org/10.33421/inmp.2019163

Recibido: 26-09-2019 Aceptado: 30-09-2019 


\section{INTRODUCCIÓN}

La violencia ejercida contra la mujer es un problema muy importante y trascendente de salud pública, así como una violación flagrante de los derechos humanos de la mujer, y son un obstáculo para el desarrollo cultural, político y económico de nuestro país. Es así mismo, expresión de inequidad, discriminación y exclusión hacia las mujeres, niñas, niños, adultos mayores y personas con discapacidad ${ }^{1}$. La violencia es un comportamiento aprendido y, por consiguiente, puede ser desaprendido ${ }^{2}$, de tal manera que se respeten los derechos del individuo y se los prepares para tomar sus propias decisiones.

Según la OMS EI 35\% de las mujeres del mundo han sido víctimas de violencia física y/o sexual por su pareja o han sufrido violencia sexual por otras personas que no son su pareja $^{3}$. Las peores consecuencias para las víctimas no son sólo las marcas del cuerpo porque estas cicatrizan, las otras, las que no se ven, son las más dañinas. La violencia psicológica y el temor permanente destruyen la autoestima de toda mujer y esto la imposibilita de defenderse de su agresor o buscar una salida o solución.

Las regiones más violentas son América Latina y el Caribe y se cree que se deba a que existe una cultura machista que rige prácticamente todas las prácticas y costumbres del quehacer diario, las cuales han postergado y limitado los derechos y libertades de las mujeres y son las vulnerables a los efectos de tener un bajo nivel económico y desigualdad. En el Perú ${ }^{4}$ se encontró que el $70,8 \%$ (2015), 68,2\% (2016) y 65,4\% (2017) de las mujeres, fueron víctimas de violencia psicológica, física, sexual o económica alguna vez en su vida por su cónyuge o pareja. Asimismo, informó que la violencia psicológica fue declarada con mayor frecuencia $(64,2 \%)$, seguida por la violencia física $(31,7 \%)$ y la violencia sexual $(6,6 \%)$.

El Ministerio de la Mujer y Poblaciones Vulnerables (MIMP), cuenta con el "El Programa Nacional Contra la Violencia Física y Sexual-PNCVF" el cual sirvió de base para la creación de los "Centros de Emergencia Mujer (CEM)" con el objetivo de brindar apoyo psicológico, físico y legal a las mujeres violentadas. Según sus reportes, en Tacna el 35,2\% (2015), el 33,4\% (2016) y el 26,9\% (2017) de las mujeres fueron víctimas de violencia psicológica, física y/o sexual, alguna vez en su vida ${ }^{5}$.

En la región Tacna, se ubican cuatro CEM regulares en Candarave, Jorge Basadre, Tacna y Tarata. Además de 2 CEM comisarías que se encuentran en Ciudad Nueva y Gregorio Albarracín. Sumando en total, seis CEM para atender los casos de violencia familiar y sexual.

Según reportes del CEM en Tacna, hasta mayo del 2018, se registraron 1047 casos por violencia familiar y estas denuncias son, en su mayoría, por maltratos físicos o psicológicos y agresiones contra las mujeres ${ }^{5}$.
Es evidente que con el paso de los años, la población presenta una menor tolerancia frente a los hechos de violencia, lo cual se hace visible con el hecho de que cada vez son más las personas que denuncian a sus agresores, es por ello que nos planteamos la siguiente interrogante: ¿Cuál es la relación entre los determinantes sociodemográficos y la violencia contra la mujer en Tacna?

En Tacna hasta la fecha, no se han publicado estudios que indiquen los factores que influyen como causalidad de la violencia contra la mujer, es por ello que en este contexto el presente trabajo de investigación pretende determinar la relación de los factores sociodemográficos y la violencia contra la mujer en los años 2015 al 2017, con el fin de poder aportar con planteamientos de intervención y recomendaciones que contribuyan a revertir este problema tan importante en nuestra sociedad, ya que a la fecha las intervenciones que se están dando a los casos de denuncia, no se evidencian resultados positivos de las actividades preventivas específicas de causalidad.

\section{MATERIALES Y MÉTODOS}

El presente estudio fue de tipo descriptivo analítico, donde se investigó a la Mujer que sufre violencia y que realizó la denuncia y/o búsqueda de ayuda en los Centros de Emergencia Mujer de la provincia de Tacna entre los años 2015 a 2017. La muestra fue depurada considerando los criterios de inclusión: Mujeres que tengan ficha de registro de casos de denuncia en el CEM y exclusión: Casos sin fichas de registro en el CEM, por desistimiento de denuncia, Casos que sean de otras jurisdicciones, provincia o departamento, y datos incompletos por mala data o contradictoria, fueron en el año 2015: 410 casos, el año 2016: 434 casos y el año 2017: 1101 casos, cuya suma total son 1945 casos, siendo la selección por conveniencia. Se contó con la autorización del Ministerio de la Mujer y poblaciones vulnerables y el Programa nacional de Violencia contra la mujer, para el acceso a la base de datos, de los diferentes CEM de la provincia de Tacna. El instrumento aplicado tuvo como base la Ficha de registro de casos del Centro Emergencia Mujer del Programa Nacional contra la Violencia familiar y sexual la cual ya ha sido validada por el MIMP, que abordan las características sociodemográficas de las agredidas y agresores, las actitudes frente a comportamientos violentos y frente a las normas, las habilidades para emitir respuestas alternativas a la violencia, la percepción de las instituciones sociales y de gobierno, el consumo excesivo de alcohol, el uso de medios de comunicación y la prevalencia de comportamientos violentos.

Para el procesamiento y análisis de datos, se diseñó una hoja de cálculos en Excel 2013, luego se procesaron en SPSS 21, que permitió la elaboración de tablas simples, de doble entrada y gráficos. Se utilizó la estadística descriptiva como: frecuencia, porcentaje. Para el análisis de los determinantes asociados se utilizó el OR (razón 
de prevalencias) con intervalos de confianza, y prueba estadística no paramétrica chi- cuadrado a fin de probar si existe o no asociación y diferencias estadísticas, considerándose un valor $p$ significativo menor de 0,05.

\section{RESULTADOS}

En la Tabla 1 podemos observar que, de la tipificación encontrada la violencia psicológica ocupa el primer lugar, seguida de la violencia física, sexual y económica.

Para el análisis de los determinantes de la violencia contra la mujer se estudiaron 4: edad, estado civil, nivel educativo y trabajo remunerado. Este análisis se realizó desde la perspectiva de la agredida y del agresor. Siendo las formas de agresión más frecuentes: los gritos e insultos, indiferencia, rechazo, desvalorización y la humillación.
En la tabla 2 la valoración del riesgo para la integridad personal y para la vida de la agredida es moderada con el $62,8 \%$. En la Tabla 3 se observa que los determinantes sociodemográficos de la agredida asociados a la violencia psicológica son: edad $(p=0,000)$ y estado civil $(p=0,000)$. Asociados a la violencia física son: trabajo remunerado $(p=0,001)$. Asociado a la violencia sexual: la edad de la agredida $(p=000)$, siendo un determinante encontrado prioritariamente en menores de 19 años; además del estado civil $(p=0,000)$. En la tabla 4 se observan que los determinantes sociodemográficos del agresor asociadas a la violencia psicológica fueron: la edad del agresor $(p=0,000)$ y sexo del agresor $(0,002)$. Asociados a la violencia física son: Edad del agresor ( $p$ : 0.000) donde las edades intermedias muestran mayor agresividad; y convivencia con la agredida $(p=0,000)$. Asociados a la violencia sexual: edad del agresor $(p=0,000)$, sexo del agresor $(p=0,000)$ y convivencia con la agredida $(p=0,000)$.

Tabla 1. Tipos de violencia contra la mujer en la provincia de Tacna según año de reporte 2015-2017

\begin{tabular}{|c|c|c|c|c|c|c|}
\hline & & \multicolumn{4}{|c|}{ Tipo de Violencia } & \multirow[b]{2}{*}{ Total } \\
\hline \multicolumn{2}{|c|}{ Fecha de Ingreso } & Violencia & Violencia & Yioloncia Fícica & Violancia Serual & \\
\hline \multirow{6}{*}{$\begin{array}{l}\text { Fecha } \\
\text { de } \\
\text { ingreso }\end{array}$} & \multirow{2}{*}{2015} & 0 & 191 & 161 & 58 & 410 \\
\hline & & $0,0 \%$ & $46,6 \%$ & $39,3 \%$ & $14,1 \%$ & $100,0 \%$ \\
\hline & \multirow{2}{*}{2016} & 0 & 218 & 162 & 54 & 434 \\
\hline & & $0,0 \%$ & $50,2 \%$ & $37,3 \%$ & $12,4 \%$ & $100,0 \%$ \\
\hline & \multirow{2}{*}{2017} & 5 & 452 & 514 & 130 & 1101 \\
\hline & & $0,5 \%$ & $41,1 \%$ & $46,7 \%$ & $11,8 \%$ & $100,0 \%$ \\
\hline \multirow{2}{*}{\multicolumn{2}{|c|}{ Total }} & 5 & 861 & 837 & 242 & 1945 \\
\hline & & $0,3 \%$ & $44,3 \%$ & $43,0 \%$ & $12,4 \%$ & $100,0 \%$ \\
\hline
\end{tabular}

Tabla 2. Valoración de riesgo para la integridad de la agredida en la provincia de Tacna según año de reporte 2015-2017

\begin{tabular}{|c|c|c|c|c|c|}
\hline & & \multicolumn{3}{|c|}{ Fecha de Ingreso } & \multirow{2}{*}{ Total } \\
\hline & & 2015 & 2016 & 2017 & \\
\hline \multirow{9}{*}{$\begin{array}{l}\text { Valoración del riesgo } \\
\text { para la integridad } \\
\text { personal y para la } \\
\text { vida }\end{array}$} & \multirow{4}{*}{ Leve } & 42 & 36 & 442 & 520 \\
\hline & & $8,1 \%$ & $6,9 \%$ & $85,0 \%$ & $100,0 \%$ \\
\hline & & $10,2 \%$ & $8,3 \%$ & $40,1 \%$ & $23,7 \%$ \\
\hline & & 344 & 350 & 527 & 1221 \\
\hline & \multirow[t]{2}{*}{ Moderado } & $28,2 \%$ & $28,7 \%$ & $43,2 \%$ & $100,0 \%$ \\
\hline & & $83,9 \%$ & $80,6 \%$ & $47,9 \%$ & $62,8 \%$ \\
\hline & \multirow{3}{*}{ Alto } & 24 & 48 & 132 & 204 \\
\hline & & $11,8 \%$ & $23,5 \%$ & $64,7 \%$ & $100,0 \%$ \\
\hline & & $5,9 \%$ & $11,1 \%$ & $12,0 \%$ & $10,5 \%$ \\
\hline \multirow{3}{*}{\multicolumn{2}{|c|}{ Total }} & 410 & 434 & 1101 & 1945 \\
\hline & & $21,1 \%$ & $22,3 \%$ & $56,6 \%$ & $100,0 \%$ \\
\hline & & $100,0 \%$ & $100,0 \%$ & $100,0 \%$ & $100,0 \%$ \\
\hline
\end{tabular}


Tabla 3. Análisis de los determinantes de la agredida asociados a los tipos de violencia contra la mujer Tacna 2015-2017

\begin{tabular}{|c|c|c|c|c|c|c|c|c|c|}
\hline \multirow{2}{*}{\multicolumn{2}{|c|}{ TIPO DE VIOLENCIA }} & \multirow{2}{*}{ B } & \multirow{2}{*}{ E.T. } & \multirow{2}{*}{ Wald } & \multirow{2}{*}{ gl } & \multirow{2}{*}{ Sig. } & \multirow{2}{*}{$\begin{array}{c}\operatorname{Exp}(B) \\
\text { OR }\end{array}$} & \multicolumn{2}{|c|}{ I.C. $95 \%$ para EXP (B) } \\
\hline & & & & & & & & inferior & Superior \\
\hline \multirow{4}{*}{ PSICOLÓGICA } & EDAD & .202 & .038 & 27.967 & 1 & .000 & 1.224 & 1.135 & 1.319 \\
\hline & ESTADO CIVIL & .154 & .034 & 20.443 & 1 & .000 & 1.166 & 1.091 & 1.247 \\
\hline & NIVEL EDUCATIVO & .032 & .023 & 1.904 & 1 & .168 & 1.032 & .987 & 1.080 \\
\hline & TRABAJO REMUNERADO & -.044 & .105 & .176 & 1 & .675 & .957 & .779 & 1.175 \\
\hline \multirow{4}{*}{ FÍSICA } & EDAD & .069 & .037 & 3.413 & 1 & .065 & 1.071 & .996 & 1.152 \\
\hline & ESTADO CIVIL & -.045 & .034 & 1.732 & 1 & .188 & .956 & .895 & 1.022 \\
\hline & NIVEL EDUCATIVO & .013 & .023 & .317 & 1 & .573 & 1.013 & .969 & 1.059 \\
\hline & TRABAJO REMUNERADO & .341 & .104 & 10.759 & 1 & .001 & 1.407 & 1.147 & 1.726 \\
\hline \multirow{4}{*}{ SEXUAL } & EDAD & -.9115 & .104 & 77.882 & 1 & .000 & .401 & .327 & .491 \\
\hline & ESTADO CIVIL & -.441 & .101 & 18.922 & 1 & .000 & .643 & .527 & .785 \\
\hline & NIVEL EDUCATIVO & .077 & .047 & 2.695 & 1 & .101 & 1.080 & .985 & 1.184 \\
\hline & TRABAJO REMUNERADO & -.445 & .213 & 4.384 & 1 & .036 & .641 & .423 & .972 \\
\hline
\end{tabular}

Tabla 4. Análisis de los determinantes del agresor asociados a los tipos de violencia contra la mujer Tacna 2015-2017

\begin{tabular}{|c|c|c|c|c|c|c|c|c|c|}
\hline \multirow{2}{*}{\multicolumn{2}{|c|}{ TIPO DE VIOLENCIA }} & \multirow{2}{*}{ B } & \multirow{2}{*}{ E.T. } & \multirow{2}{*}{ Wald } & \multirow{2}{*}{ gl } & \multirow{2}{*}{ Sig. } & \multirow{2}{*}{$\begin{array}{c}\operatorname{Exp}(B) \\
\text { OR }\end{array}$} & \multicolumn{2}{|c|}{ I.C. $95 \%$ para EXP (B) } \\
\hline & & & & & & & & inferior & Superior \\
\hline \multirow{3}{*}{ PSICOLÓGICA } & EDAD DEL AGRESOR & .321 & .041 & 60.376 & 1 & .000 & 1.378 & 1.271 & 1.495 \\
\hline & SEXO DEL AGRESOR & -.541 & .171 & 10.054 & 1 & .002 & .582 & .417 & .813 \\
\hline & $\begin{array}{l}\text { CONVIVENCIA CON LA } \\
\text { AGREDIDA }\end{array}$ & .085 & .088 & .929 & 1 & .335 & 1.088 & .916 & 1.293 \\
\hline \multirow{3}{*}{ FÍSICA } & EDAD DEL AGRESOR & -.207 & .041 & 25.303 & 1 & .000 & .813 & .750 & .881 \\
\hline & SEXO DEL AGRESOR & .027 & .172 & .025 & 1 & .875 & 1.027 & .734 & 1.439 \\
\hline & $\begin{array}{l}\text { CONVIVENCIA CON LA } \\
\text { AGREDIDA }\end{array}$ & -.600 & .089 & 45.186 & 1 & .000 & .549 & .461 & .654 \\
\hline \multirow{3}{*}{ SEXUAL } & EDAD DEL AGRESOR & -.289 & .065 & 19.370 & 1 & .000 & .751 & .662 & .853 \\
\hline & SEXO DEL AGRESOR & 2.146 & .590 & 13.236 & 1 & .000 & 8.549 & 2.691 & 27.160 \\
\hline & $\begin{array}{l}\text { CONVIVENCIA CON LA } \\
\text { AGREDIDA }\end{array}$ & 1.165 & .143 & 66.299 & 1 & .000 & 3.205 & 2.421 & 4.242 \\
\hline
\end{tabular}

\section{DISCUSIÓN}

Los principales hallazgos del estudio indican, que La violencia psicológica es la predominante. Resultados que muestran similitud con publicados por el INEI a nivel nacional, el $61,5 \%$ denuncia por violencia psicológica y/o verbal, el $30,6 \%$ violencia física y el $6,5 \%$ por violencia sexual ${ }^{4}$; existiendo diferencias locales y regionales, como en Junín donde la violencia física supera a la psicológica. Estos resultados nos llevan a realizar necesariamente un análisis y tomar alerta, ya que en la actualidad vemos con mucha preocupación que la violencia física se incrementa y acorta la brecha con el feminicidio que es el resultado más grave de violencia contra la mujer.

Los determinantes de la agredida asociados a violencia psicológica, física y sexual fueron: edad, estado civil, condición de trabajo remunerado y el nivel educativo. A mayor edad mayor probabilidad violencia psicológica, entre 20 y 49 años mayor riesgo de violencia física, menor 19 años mayor riesgo de violencia sexual. La condición de separada, divorciada, ex convivientes y el haber tenido antes una pareja, se convierte en un determinante de riesgo asociado a la violencia. También a mayor grado de instrucción las probabilidades de violencia psicológica son mayores. Dato que difiere de lo encontrado en el estudio de Fiesta ${ }^{6}$, quien señala que las mujeres que tienen educación universitaria tuvieron menor probabilidad de ser víctimas de violencia psicológica, lo cual nos permite evidenciar como en el contexto social actual el nivel de instrucción ya no es un factor de protección para evitar la violencia contra la mujer. En nuestro estudio respecto a que las víctimas cuentan en su mayoría con un trabajo remunerado, dato que difieren con ChavarriaMejia $\mathrm{J}$ et $\mathrm{al}^{7}$ donde la mujer víctima de violencia tiene como riesgo el ser ama de casa $(38,84 \%)$ y sin ingreso económico $(59,50 \%)$, deja en claro que la sociedad vive una crisis respecto a la inteligencia emocional del hombre 
donde ahora ve a la mujer con desconfianza y como su competencia. Sin embargo, nuestros hallazgos son similares a la investigación de Díaz y Miranda ${ }^{8}$, donde las mujeres que cuentan con un trabajo remunerado tienen mayores probabilidades de ser agredidas, más aún cuando el hombre está desempleado, o cuando la mujer gana más dinero que su pareja.

Es importante mencionar que la violencia física cuando es conyugal acrecienta la violencia familiar, provocando un cambio negativo en la autoestima de los integrantes involucrados de manera directa o indirecta, lo que muchas veces repercute en la distribución de los recursos económicos en forma irracional generando dependencia y sometimiento a la mujer. Generando una dependencia emocional de las víctimas para realizar las denuncias respectivas como indican en los resultados de la investigación de Tello": "una mujer con dependencia emocional experimentará elevados niveles de miedo al pensar que su relación de pareja pueda terminar" y como dice Castelló (2005), "la sumisión representa para la persona dependiente un medio para que la relación no termine, puesto que hay miedo a la ruptura, que en última instancia revela esa fuerte necesidad de vinculación afectiva a la pareja que es el sustento de la dependencia emocional".

Respecto a los determinantes sociodemográficos del agresor asociadas a la violencia psicológica, física, sexual y económica, la edad, el sexo del agresor y la convivencia son altamente influyentes. Las mujeres agresoras tienen de 30 a 39 años mayormente similar a la edad de los hombres; sin embargo solo el $8 \%$ son agresoras y el $92 \%$ agresores, con respecto al vínculo con la agredida, la agresora tiene el rol de madre en un $15,8 \%$ de los casos y un $57,6 \%$ es desconocida y/o no tiene vínculo familiar, lo que se evidencia cuando analizamos la ocupación de la mujer agresora, que en su mayoría es comerciante y ambulante y tiene exposición a enfrentamiento por la actividad misma que desarrolla, generando ello agresión de mujer a mujer. Cuando el agresor es varón, el resultado es por la condición de ser el conviviente, ex conviviente y ser actualmente el esposo. Con respecto a la violencia física la edad del agresor (entre 20 a 49 años) y convivencia con el agresor en la misma casa de la víctima son determinantes de violencia. Datos que difieren de los hallazgos encontrados por Nóblega ${ }^{10}$, donde sus resultados muestran que no hay asociación estadística significativa entre la violencia física y psicológica contra la mujer y la edad $(r=0,098$ y $r=0,021$ respectivamente para cada tipo de violencia).

Respecto a los determinantes del agresor asociados a la violencia sexual, encontramos existe asociación con la edad, donde la mayor frecuencia se presentó en edades adultas; con el sexo del agresor, donde se evidencia que los hombres son más agresores, con una estadística altamente significativa; con la convivencia con la agredida. Las tres variables exploradas se tienen una asociación estadística altamente significativa. $(p=0,000)$. De las principales formas de violencia sexual, encontramos que la violación sexual es la frecuente $(n=128)$, actos contra el pudor $(n=73)$ y acoso sexual $(n=14)$. Con una significancia estadística $\quad(p=0,000)$.

Estos resultados nos permiten afirmar que las condiciones sociodemográficas de los agresores son un elemento que posibilita discriminar entre las mujeres que sufren violencia física o psicológica y quiénes no.

Consideramos que crecer en un contexto familiar violento, permite predecir la aparición de problemas emocionales, cognitivos y conductuales a corto o largo plazo, niños que son testigos de situaciones de violencia, los convierte en potencial factor de riesgo, para repetir la pauta conductual en pareja cuando crezcan; y en el caso de las niñas las convierte en mujeres que asumen un papel pasivo de aceptación de la violencia. Por ello la familia es la clave en el desarrollo de culturas y civilizaciones, y fundamental en el desarrollo integral de la sociedad; las familias que se forman saludables, fuertes y sostenibles generan una sociedad fuerte, saludable y sostenible. Por el contrario, cuando hay un debilitamiento o ruptura de la estructura y dinámica familiar, ésta repercute negativamente en la sociedad, causando problemas entre sus miembros. Según Ignasi Bofarull del instituto de estudios superiores de la familia Universidad de Cataluña indica que la familia es fundamental en el desarrollo integral de la sociedad.

Es importante mencionar algunas limitaciones del presente estudio. Una de ellas es que la fuente de análisis de información de los CEM-TACNA para los años de estudio no contaba con variables similares en su totalidad, por esta razón, por su complejidad, la manera de medirla no incluye otros tipos de violencia como la económica en los tres años de estudio. Para un mayor análisis y poder establecer mayor causalidad en la violencia contra la mujer, se sugiere el desarrollo de investigaciones prospectivas con metodologías mixtas cuantitativascualitativas, con participación de agredidas y agresores.

En conclusión, este estudio nos proporciona evidencia consistente que existen determinantes sociodemográficos con mayor probabilidad que están asociados a la violencia contra la mujer. a mayor edad mayor riesgo de violencia psicológica, edades entre 20 y 49 años mayor riesgo de violencia física, <19 años mayor riesgo de violencia sexual. Tener trabajo remunerado aumenta el riesgo de violencia.

Determinantes del agresor predominantes son: edad con características similares para la violencia psicológica, física y sexual que, en la mujer, con evidencia que la mujer agrede a otra mujer.

Esta información puede ayudar a generar programas de intervención o políticas públicas que tengan la finalidad de reducir la violencia contra la mujer y sobre todo disminuir 
efectos negativos en la salud de las mujeres víctimas de daños producidos por la violencia psicológica, física y sexual. Así, mismo consideramos que es fundamental la atención de los resultados ya que el enfoque preventivo es muy importante; vemos con preocupación que los programas y políticas están orientados a la consecuencia de la violencia contra la mujer con prioridad. También debemos de tomar en cuenta que las intervenciones deben orientarse en las familias de nuestra sociedad.

\section{AGRADECIMIENTOS}

Queremos expresar un profundo agradecimiento a las Autoridades de la Universidad Nacional Jorge Basadre Grohamnn, Vice rectorado de investigación, como el órgano director de la actividad de investigación científica, tecnológica y humanística del más alto nivel de la Universidad. Al Ministerio de la Mujer y Poblaciones Vulnerables (MIMP) como ente rector de las políticas nacionales y sectoriales sobre derechos de las mujeres, al Programa Nacional Contra la Violencia Familiar y Sexual (PNCVFS), que nos brindaron las facilidades para el desarrollo de la presente investigación.

Financiamiento: Ministerio de la Mujer y Poblaciones Vulnerables-Programa Nacional contra la violencia familiar y sexual: quienes nos brindaron acceso a la base de datos con los casos de registrados de violencia.

La investigación fue promovida por el vicerrectorado de Investigación de la Universidad Nacional Jorge Basadre Grohmann de Tacna.

Conflicto de interés: Los autores declaran no tener algún conflicto de intereses.

\section{REFERENCIAS BIBLIOGRÁFICAS}

1. Guia-de-atención-de-los-CEM.pdf [Internet]. [citado 18 de abril de 2019]. Disponible en: https://www.mimp.gob.pe/files/ programas_nacionales/pncvfs/stapas/GUIA-DE-ATENCIONDE-LOS-CEM.pdf
2. Velzeboer M. La violencia contra las mujeres: responde el sector de la salud. Organizacion Panamericana de la Salud; 2003. book.

3. Secretario General de las Naciones Unidas, Ban Ki-Moon (2008) lo que dijo acerca la violencia. - Buscar con Google [Internet]. [citado 21 de marzo de 2019]. Disponible en: https://www.google.com/search?ei=wF2uXPTLKeTO5gKAkq mIBQ\&q=Secretario+General+de+las+Naciones+Unidas $\% 2$ C+Ban+Ki-Moon+\%282008\%29+lo+que+dijo+acerca+la+vio lencia.\&oq

4. INEI. Indicadores de violencia familiar y sexual 2000-2017. Perú. 2017 [Internet]. [citado 10 de abril de 2019]. Disponible en: $\quad$ https://www.inei.gob.pe/media/MenuRecursivo/ publicaciones_digitales/Est/Lib1465/libro.pdf

5. Ministerio de la Mujer y Poblaciones Vulnerables - MIMP [Internet]. [citado 10 de marzo de 2019]. Disponible en: https://www.mimp.gob.pe/contigo/contenidos/pncontigoarticulos.php?codigo $=8$

6. Fiestas F, Rojas R, Gushiken A, Gozzer E. ¿Quién es la víctima y quién el agresor en la violencia física entre parejas? Estudio epidemiológico en siete ciudades del Perú. Rev Peru Med Exp Salud Pública. 2012;29(1):44-52.

7. Chavarria J, Calix D, Vasquez W. Caracterización de violencia doméstica en mujeres mayores de 16 años con compañero en comunidad de Honduras 2016. CIMEL. 2017;22(1):32-39.

8. Díaz R, Miranda JJ. Aproximación del costo económico y determinantes de la violencia doméstica en el Perú :91.

9. Tello JJA. Dependencia emocional en mujeres víctimas de violencia de pareja. Rev Psicol. 2015;33:26.

10. Nóblega M. Características de los agresores en la violencia hacia la pareja. Liberabit. 2012;18(1):59-68.

Correspondencia: Olga Choque Chura.

Dirección: Av Miraflores S/N cercado de Tacna (Ciudad universitaria de la UNJBG).

Correo: Olgirasol16@hotmail.com

Teléfono: 952229510 